\title{
Adolescentes e crianças vítimas de violência atendidas em um Programa de Psicologia em Delegacias da Mulher do ABC Paulista*
}

\section{Adolescents and children victims of violence served in a psychology program in Women's police station of Brazil}

\author{
Amanda Munari** \\ Rafaela Perricone ${ }^{* *}$ \\ LARISSA SMERDEL *** \\ JULIANA OLIVEIRA *** \\ Marília M. VizzotTo ${ }^{* * * *}$
}

\begin{abstract}
Resumo
Este estudo levantou dados de prontuários de clientela (adolescentes e crianças) que recebera assistência em um "programa de atenção psicológica imediata às vítimas de violência", em delegacias especiais para mulheres e crianças, localizadas na região da grande São Paulo, Brasil. Entre os 354 registros de atendimentos psicológicos nos anos de 2014 a 2016, as crianças e adolescentes totalizaram 32. Desse total, 20 registros (62\%) eram de vítimas do sexo feminino, na faixa etária de 4 a 16 anos. Verificou-se que as principais queixas sobre violência
\end{abstract}

\footnotetext{
* Este trabalho foi originalmente apresentado em "XXI Congreso Nacional de Psicodianostico - ADEIP" (2017), Buenos Aires, Argentina.

* Acadêmicas do Curso de Psicologia.

*** Psicólogas, graduadas pelo Curso de Psicologia da Universidade Metodista de São Paulo.

**** Doutora pela Faculdade Ciências Médicas UNICAMP - Universidade Estadual de Campinas/ Professora orientadora da pesquisa.
} 
(Abuso sexual; agressões física/verbal; negligência) não se distanciaram dos dados apresentados pelos órgãos oficiais e pelas pesquisas. A violência praticada contra crianças esteve sempre relacionada ao contexto familiar. É possível considerar a família como eixo central de atenção sendo ela o grande foco da prevenção.

Palavras-chave: Violência Infantil; abuso sexual; agressão física; atenção psicológica

\section{Abstract}

This study has collected data on patients' charts (adolescents and children) who received assistance in a "program of immediate psychological attention to victims of violence" in police stations in Brazil. Of the 354 records of violence victims (years 2014 to 2016), children and adolescents totaled 32 . Of this total, 20 records (62\%) were of female victims, in the age group of 4 to 16 years. The main complaints about violence (sexual abuse, physical / verbal aggression, neglect) were not distanced from the data presented by the official bodies and the investigations. Violence against children has always been related to the family context. It is possible to consider the family as the central focus of attention and it is the main focus of prevention.

Keywords: Child Violence; sexual abuse; Physical aggression; psychological attention

\section{Violencia: América Latina, Brasil}

O Brasil tem uma das legislações mais avançadas do mundo quando se trata da proteção de crianças e adolescentes, haja vista a instituição do Estatuto da Criança e do Adolescente (ECA), aprovado em 1990 e que estabelece a responsabilidade de proteção integral das crianças e adolescentes até os 18 anos (maioridade) à sociedade e ao Estado. A própria Unicef - Fundo das Nações Unidas para a Infância (UNICEF, 2017) anunciou em relatório que o Brasil é um dos 59 países que têm legislação que proíbe castigo físico e apenas $9 \%$ das crianças com menos de 5 anos em todo o mundo vivem nesses países, o que deixa outros 607 milhões sem uma proteção legal contra esse tipo de violência. Todavia, como afirmou Francischini (2003) essas determinações legais estão longe de efetivação na prática; isto, pois a própria desproteção, parece ser reforçada, muitas 
vezes, pela passividade da sociedade, que convive com a observação de índices alarmantes de atos violentos.

O Brasil está entre os seis países mais violentos da América Latina com as maiores taxas de homicídio, sendo o quinto em homicídios juvenis, revela estudo do Instituto Igarapé. O UNODC (2013) mostrou que em 2012 os países com maiores taxas de homicídios na América Latina, foram Honduras, seguido por Venezuela, Belize, El Salvador, Guatemala e Colômbia. E entre os que tinham taxas abaixo de 10 homicídios por 100.000 habitantes, estiveram o Paraguai, Peru, Costa Rica, Uruguai, Suriname e Chile. E, se no ano de 2013 os pesquisadores já consideravam altas essas taxas, o que se constata em 2018 pelo levantamento do "Atlas da Violência" é ainda mais amedrontador, pois o relatório produzido pelo Ipea e pelo Fórum Brasileiro de Segurança Pública (FBSP), em que se analisou inúmeros indicadores para compreender acentuada violência no país, encontra-se os seguintes dizeres : "Neste momento, no Brasil, a taxa de homicídios ultrapassa a marca nunca antes vista de 30 por 100 mil habitantes" (IPEA; FBSP, 2018, p. 89).

Ante a um quadro crescente e preocupante, encontram-se também as formas de violência doméstica e especialmente aquelas contra as crianças e adolescentes. A Unicef (2017) traz um relatório sobre as diversas formas de violência que crianças e adolescentes sofrem em todo o mundo: violência disciplinar e violência doméstica na primeira infância; violência na escola - incluindo bullying; violência sexual; e mortes violentas de crianças e adolescentes. Indica que, entre outros dados, três quartos das crianças de 2 a 4 anos do mundo - cerca de 300 milhões - sofrem agressão psicológica e/ou punição física tendo como autores os seus cuidadores. A América Latina e o Caribe concentram cerca da metade de homicídios de crianças e adolescentes em todo o mundo; números se mostram desproporcionais considerando que tal conjunto de países abriga pouco menos de $10 \%$ da população nessa faixa etária. Ainda dados da própria Unicef (2018) indicaram que na América latina e Caribe são encontrados que cerca de 85 mil crianças morrem anualmente em decorrência da violência doméstica; a violência atinge, aproximadamente, 6 milhões de meninas e meninos. 
Além dos órgãos oficiais, outros estudos têm sido feitos em várias regiões do país e, do mesmo modo, o cenário é bastante inquietante. Diferentes variáveis têm sido associadas ao fenômeno da violência infantil. A prevalência de um dos sexos em violência doméstica e abuso sexual, como indicaram Cohen, (1993); Saffioti, (1997); Habigzang, Koller, Azevedo e Machado (2005); Farias, Souza, Carneseca, Passos e Vieira (2016) mostram a prevalência de crianças do sexo feminino em quase todas as faixas etárias. No que se refere aos homicídios há, segundo Melo, Silva e Garcia (2017) risco de morte por agressões em jovens do sexo masculino, mas isso está intimamente relacionado às características sociodemográficas como os grandes municípios, mais urbanizados e com maior proporção de jovens buscando emprego e fora do ensino médio. Já Pinto Jr, Cassepp-Borges e Santos (2015) apontaram para as variáveis renda e escolarização familiares baixas associadas à vitimização infantil/ adolescente. Minayo (2001) já havia apontado para a desigualdade social, num quadro que tem sido associado às agressões dentro das próprias famílias. Do mesmo modo que a Unicef $(2017 ; 2018)$, Peres, Ruotti, Carvalho e Regina (2015) indicaram que a violência ocorre em todo o mundo frequentemente em regiões mais pobres.

A violência doméstica contra crianças e adolescentes é um fenômeno amplo e complexo, que se origina de diferentes fatores e tem consequências devastadoras para as vítimas; de modo que, estudá-lo mais, sempre se faz relevante.

Assim, o objetivo deste estudo foi levantar dados de prontuários sobre da clientela (adolescentes e crianças) que recebera assistência em um programa de atenção psicológica imediata às vítimas, em delegacias especiais para mulheres e crianças, localizadas na região de São Paulo, Brasil.

\section{Método}

Este se trata de estudo exploratório, descritivo e documental, com abordagem quantitativa, que caracterizou casos de violência doméstica contra crianças e adolescentes. Os dados foram tratados a partir de registros arquivados no Curso de Psicologia; esses registros são prontuários de pessoas atendidas (atendimentos realizados por estudantes de psicologia - $5^{\circ}$. ano de graduação) em um programa 
de 'atendimento imediato' (ou plantões) realizado nas instalações das próprias Delegacias da Mulher - da região da grande São Paulo (ABCD paulista e regiões da capital deste entorno - Artur Alvim, Vila Carrão). Os registros foram analisados individualmente, caso a caso pelos pesquisadores e, em seguida, foram feitas as categorias de análise. Após, foram feitas estatísticas descritivas. Entre os 354 prontuários existentes nos arquivos da clínica psicológica da Universidade Metodista dos anos de 2014 a 2016, os registros de crianças e adolescentes totalizaram 32. Destacou-se que, desse total, 20 prontuários (62\%) eram de pessoas do sexo feminino e 12 do sexo masculino, em faixa etária de 4 a 16 anos. Esse estudo atendeu a todos os princípios éticos preconizados em pesquisas envolvendo seres humanos, garantindo o sigilo e a confidencialidade dos dados coletados. O projeto foi submetido à análise do Comitê de Ética em Pesquisa da Universidade Metodista de São Paulo.

\section{Resultados e discussão}

Analisaram-se 32 prontuários de casos de crianças e adolescentes atendidos pelos plantonistas em psicologia em um programa de atenção psicológica a vitimas de violência doméstica. Foram observados que, em todos os casos, as crianças e os adolescentes moravam em casas com suas famílias, tinham baixo rendimento econômico e, embora freqüentassem escolas, seus familiares tinham baixa escolaridade. Esses dados coincidem com Pinto Jr, Cassepp-Borges e Santos (2015) em um levantamento de dados realizado em Volta Redonda, R.J, em que observaram percentual elevado de famílias que viviam com até um salário mínimo, e as crianças vitimizadas freqüentavam escolas; e seus agressores (predominantemente seus pais) tinham baixa escolaridade.

A escolarização baixa associada à violência tem sido, entre outras variáveis, um fator de muitas discussões. Já no início do ano dois mil, Minayo (2001) lembrou os dados do próprio IBGE - Instituto brasileiro de geografia e estatítisca sobre renda e escolarização da população e a autora apontou para o que chamou de 'violência estrutural', indicando que essa é uma forma de expressão de violência de crianças e adolescentes brasileiras de 0 a 17 anos, e que, ainda no ano 2000 se encontravam em situação de pobreza, vivendo 
em famílias com renda média mensal de $1 / 2$ salário mínimo per capita. Essa precariedade leva à persistência da desigualdade social, e esse quadro tem sido associado às agressões dentro das próprias famílias. Além das famílias, como um ciclo que vai se formando, os diferentes tipos de violência vão tomando um contorno mais abrangente e, como apontam Peres, Ruotti, Carvalho e Regina (2015) a violência ocorre em todo o mundo, mas é mais frequente em regiões mais pobres, contextos caracterizados por superpopulação, pobreza, altos índices de desemprego, padrões educacionais e de moradia baixos e ausência de equipamentos sociais.

É então interessante observar estudo da área econômica (BECKER; KASSOUF, 2017) que analisou gastos públicos em educação e redução de taxa de homicídios no Brasil. Autores utilizaram dados socioeconômicos dos estados brasileiros e um modelo de painel dinâmico com uma defasagem para os gastos com educação por habitante e seus resultados mostraram uma elasticidade negativa de aproximadamente 0,1 na primeira defasagem, ou seja, os gastos com educação ao aumentarem $10 \%$, a taxa de homicídios diminuía $1 \%$ no período seguinte; dados que mostraram que investimentos em educação podem ser uma forma de política pública de longo prazo para reduzir a criminalidade.

\section{Caracterização segundo o sexo}

O sexo feminino prevaleceu (62\%) dos casos atendidos eram de meninas. Estes dados corroboram com levantamentos anteriores e outros estudos (BRANCALHONE; FUEGO; WILLIAMS, 2004) em que se observou que as mulheres são as vítimas preferenciais das agressões na família. Ainda que alguns autores (ARAÚJO, 2002, ONU, 2006) discutam que as violências física, psicológica e sexual ocorrem na família e ocorrem independentemente do sexo da criança, Cohen, (1993); Saffioti, (1997) indicaram que as meninas sofrem numa porcentagem muito maior do que quando comparadas com meninos, sendo o mesmo fora observado por Pinto Jr, Cassepp-Borges e Santos (2015) em que vítimas de violência doméstica majoritariamente eram do sexo feminino, concentrandas nas faixas dos 14 aos 18 anos. Habigzang, Koller, Azevedo e Machado (2005) observaram que as meninas são vítimas de abuso sexual em maior 
frequencia quando comparados com os meninos. Farias, Souza, Carneseca, Passos e Vieira (2016) num levantamento de dados do Sistema de Vigilância de Violências e Acidentes de Ribeirão Preto encontraram que entre as vítimas de violência, prevaleceram crianças do sexo feminino (56,4\%) em quase todas as faixas etárias, exceto entre oito e nove anos de idade.

É interessante descrever que em relatório, a Organização Mundial de Saúde ${ }^{1}$ (OMS, 2014/2015) observa bem essa questão acerca da "violência e sexos" isso porque essa organização levanta o fato de que a violência fatal não está distribuída uniformemente entre grupos etários e de gênero, pois os homens correspondem a $82 \%$ de todas as vítimas de homicídio, com taxas estimadas mais de quatro vezes aquela das mulheres - 10,8; e as maiores taxas homicídio estimadas encontram-se no grupo de homens entre 15 e 29 anos de idade $-18,2$ por cem mil. Mais adiante o mesmo relatório indica que, em termos de violência doméstica - no que tange a maus-tratos contra a criança indicam que $22,6 \%$ dos adultos em todo o mundo sofreram abusos físicos na infância, 36,3\% sofreram abuso emocional e $16,3 \%$ sofreram negligência física, sem diferenças significativas entre meninos e meninas (30-32). No entanto, prevalência na vida de abuso sexual na infância indica diferenças mais acentuadas por gênero - $18 \%$ para meninas e $7,6 \%$ para meninos.

\section{Caracterização segundo a idade}

As idades variaram de 04 a 16 anos ( $n=10$ anos). Essa faixa etária é a mesma já indicada por Minayo (2001). Também sobre esse aspecto Melo, Silva e Garcia (2017) consideraram importante os dados do relatório da OMS de 2015 ao analisarem e associarem as grandes variações regionais nas taxas de mortalidade por violência entre os jovens. Entre essas comparações, observou-se que o Brasil, bem como outros países da América Latina, Caribe e também da África, tem taxas de mortalidade por violência em jovens cem vezes mais altas do que aquelas em países da Europa Ocidental e do Pacífico Ocidental (regiões em que estão as mais baixas taxas do mundo).

\footnotetext{
1 Relatório traduzido e supervisionado pelo NEV - USP (Núcleo de Estudos Violência) e publicado em português em 2015
} 


\section{Caracterização de acordo com as Denúncias}

As queixas (tabela 1) são apresentadas pela criança ou adolescente - sempre acompanhadas por uma pessoa responsável (geralmente mãe ou professor).

É necessário enfatizar que o número de queixas é sempre maior que o número de pessoas, já que é comum que o mesmo indivíduo apresente mais de uma queixa associada.

Tabela 1 - queixas apresentadas por crianças e adolescentes

\begin{tabular}{l|c|c}
\hline Principais Denúncias & Número & Porcentagem \\
\hline Violência Sexual & 14 & $30,4 \%$ \\
\hline Agressão (física e verbal) & 12 & $26,1 \%$ \\
\hline Negligência & 12 & $26,1 \%$ \\
\hline Outros tipos de Violência & 08 & $17,4 \%$ \\
\hline TOTAL & 46 & $100 \%$ \\
\hline
\end{tabular}

“Violência sexual" (30,4\%) - o abuso sexual foi compreendido como o contato ou interação entre a criança/adolescente com um adulto ou pessoa em um estágio psicossexual mais avançado de desenvolvimento. São casos em que a vítima estava sendo usada para a estimulação sexual do agressor.

Estes casos mostraram que o abuso fora cometido dentro da família e, em 70\% deles estavam os pais biológicos do sexo masculino e somente uma pequena parte por padrastos e outros. Sobre o fato de que uma grande parcela de violência ser de abusos sexuais, os dados vêm ao encontro dos achados de Pinto Jr, Cassepp-Borges e Santos (2015) investigaram casos de violência em Volta Redonda ,RJ e entre as formas de violência a sexual foi uma das mais identificadas pelos pesquisadores. Esses autores entenderam que nessa forma de vitimização estavam envolvidos fatores sociais e familiares complexos (entre eles os riscos de gravidez precoce e de doenças sexualmente transmissíveis), além dos significativos agravos para a saúde das vítimas.

Já com relação ao sexo masculino, esse dado coincide com aqueles encontrados por Farias, Souza, Carneseca, Passos e Vieira (2016) 
que identificaram que entre os agressores de crianças, predominaram homens $(53,6 \%)$ e desses a violência foi perpetrada pelos pais ou por outros familiares, com destaque para a participação paterna $(22,7 \%)$. Também Santos, Costa, Amaral, Nascimento Sobrinho, Musse e Costa (2015), em levantamento de ocorrências policiais na Bahia, encontraram que a maioria dos agressores sexuais de crianças e adolescentes era do sexo masculino (98,0\%), solteiro (61,3\%), 43,6\% adulto (25-49 anos), $18,8 \%$ adolescente e $22 \%$ adulto jovem (20-24 anos). A maioria fazia parte do ciclo de amizade da vitima (vizinho, comunidade, amigo, namorado, pai ou padrasto ou outros familiares).

Entretanto, é interessante atentar para aquilo que Reis, Barros e Cavalcante (2015) discutem num amplo trabalho de levantamento de artigos publicados sobre a temática do abuso sexual; ou seja, muito se atribui ao homem esse tipo de violência, mas pouco se observa sobre a participação feminina como agressora. Os autores mostram que a retórica de que o masculino é violento por si só é estigmatizante e a ideia de que o comportamento agressivo é masculino é reforçada na coletividade e em determinadas sociedades, como no caso do Brasil. Os autores então chamam a atenção para a discussão sobre a necessidade de um olhar para os agressores sexuais, principalmente estudos que possibilitem a investigação mais aprofundada de aspectos biopsicossociais característicos dos agressores e de pesquisas empíricas de cunho qualitativo que permitam a aproximação do fenômeno.

A presente investigação aponta efetivamente para o agressor masculino, dentro da família e especificamente a participação paterna. Todavia, os questionamentos de Santos, et al (2015) parecem pertinentes desde uma análise psicológica subjetiva, em o quanto a mulher está ou não envolvida por omissão ou conivência; o que também é violência. Aspecto que merece ser estuda clinicamente ou qualitativamente.

"Negligencia" (26,1\%) - compreendida como falta de cuidado ou desatenção e implica em omissão ou falta de observação do dever.

Sobre esses dados, estudo sobre casos de negligência em Curitiba realizado por Egry, Apostólico, Albuquerque, Gessner e Fonseca (2015) identificou que os meninos sofrem mais negligência do que as meninas e que as mulheres (mães ou cuidadoras) são as principais 
responsabilizadas, sobretudo no que tange à negligência relativa à educação e à saúde. Mais da metade das negligências notificadas envolveram crianças menores de dez anos. Em 2010, observou-se uma proporção de cinco notificações de negligência para cada 100 crianças desse grupo etário.

"A gressão física" (26,1\%) compreendida como violência em que se usam a força física, maus tratos. Essa é um tipo de queixa que vem sempre muito compartilhada com as categorias "abuso sexual" e "outras formas de violência". É um aspecto que vem sendo muito associado também, pelos autores a formas de educação da criança. Castro (2017) em estudo de uma região no nordeste brasileiro mostrou taxas elevadas de prevalência de 'agressão física' com crianças pela família; a autora conclui que alguns pais continuam a entender e praticar a disciplina com atos violentos (que se traduzem em ameaça psicológica; punição corporal; maus tratos físicos; maus tratos graves). Isso ainda revela, tal como descrevem Peres, Ruotti, Carvalho e Regina (2015) que atos violentos cometidos contra as crianças, por vezes, são legitimados pelo Estado e pela sociedade, pois ocorrem sob os disfarces da "tradição" ou "disciplina".

Torna-se importante relatar, que muito embora a composição dos perpetradores seja diversa, pois inclui os pais, familiares, professores, e outras autoridades responsáveis pela aplicação da lei e até mesmo outras crianças, nas três formas de violência aqui descritas, prevaleceu o ambiente familiar e por membros da família, por pessoas aparentadas ou muito próximas da criança.

"Outros tipos de violencia" $(17,4 \%)$ compreenderam-se e incluíram a agressão verbal, o tratamento degradante, as ofensas e bullying (cometidos em escolas).

É interessante relatar que esses tipos de violência não são estranhos à sociedade e coincide com outros países em que a violência doméstica é constada como alta. Em função disso, relatório da OMS (2015) parceria para língua portuguesa com Núcleo Estudos sobre Violência - NEV - USP mostra que estudantes que praticam bullying na escola mostram-se mais propensos a se envolverem com agressões violentas mais tarde na vida.

Outra questão a ser observada e que também é mostrada no meso relatório OMS/NEV (2015) refere-se aos próprios custos trazi- 
dos à sociedade e Estado em detrimento da violência infantojuvenil. Essa violência é acompanhada por destruição de bens e de infraestrutura; há custos médicos diretos, perdas de ganhos no futuro, custos de programas públicos, danos a propriedades e à qualidade de vida. Há ainda custos judiciais e de detenção, custos de programas de tratamento e de encarceramento, e perda de rendimentos. A economia em determinada região pode ser prejudicada quando a violência se revela elevada, conduzindo a região a ter custos substanciais com cuidados de saúde e de justiça criminal.

\section{Considerações finais}

Este estudo se referiu a uma pequena amostra de prontuários de pessoas atendidas em um programa de atenção psicológica às vitimas de violência doméstica e, portanto, oferece limitações. É fato que essa pequena amostra não representa o número de registros feitos pelas delegacias da Mulher, mas sim aqueles em que constam que essas pessoas receberam atenção psicológica no local da queixa. Deste modo, mesmo sendo uma sub-amostra, tornou-se importante observar essas características para compor dados que indicam a necessidade de estatísticas sobre os casos registrados (quando são registrados).

Foi possível observar que a faixa etária (4 a 16 anos), a predominância do sexo feminino e as principais queixas sobre violência (Abuso sexual; agressões física/verbal; negligência) não se distanciam dos dados apresentados pelos órgãos oficiais e pelas pesquisas. As queixas de violência dessas pessoas atendidas estiveram sempre relacionadas ao contexto familiar; de modo que é possível considerar a família como eixo central de atenção nos casos de violência doméstica, embora isso não seja novidade; mas vale a pena corroborar que é na família que reside o grande foco da prevenção.

Assim, a descrição dessas diferentes formas de violências infantojuvenis corrobora a existência de um circulo vicioso na vida dessas, mostrando ainda que essas crianças e jovens vitimizados são pertencentes a agrupamentos sócio-familiares que as expõem a uma série de situações degradantes nos próprios ambientes em que vivem.

Também é possível dizer, que embora o Brasil tenha uma legislação moderna, faltam políticas públicas que permitam manter 
programas de proteção, prevenção e mesmo de tratamento para que contribuam para o aprimoramento de técnicas efetivas no enfrentamento desse problema.

\section{Referências}

ARAÚJO, M.F. Violência e abuso sexual na família. Psicologia em Estudo, v.7, n.2, p. 3-11, 2002.

BECKER, K.L.; KASSOUF, A.L. Uma análise do efeito dos gastos públicos em educação sobre a criminalidade no Brasil. Economia e Sociedade, Campinas, v. 26, n. 1 (59), p. 215-242, abr. 2017.

BRANCALHONE, P. G.; FOGO, J. C.; WILliAMS, L. C. A. Crianças Expostas à Violência Conjugal: Avaliação do Desempenh o Acadêmico. Psicologia: Teoria e Pesquisa, v. 20, n. 2, p.1-5, ago, 2004

CASTRO, J. V.B. Relação da Violência Doméstica com o Aproveitamento Escolar: Percepções Entre Crianças e Adolescentes, de uma Escola Municipal na Cidade de Campina Grande - Paraíba. 172 fls. (Dissertação), Instituto de Educação da Universidade Lusófona de Humanidades e Tecnologias - Lisboa, 2017

COHEN, C. O incesto. In: AZEVEDO M.A; GUERRA, V.N. (Orgs.), Infância e Violência Doméstica: fronteiras do conhecimento, p.211-225, São Paulo: Cortez, 1993.

EGRY EY, APOSTÓLICO MR, ALBUQUERQUE LM, GESSNER R, FONSECA RMGS. Compreendendo a negligência infantil na perspectiva de gênero: estudo em um município brasileiro. Rev Esc Enferm USP ;; v.49, n. 4, :p.556-563, 2015

FRANCISCHINI, R. As faces da infância em contextos de violência física intrafamiliar. Revista de Psicologia da UNESP, v. 2, n. 1, p. 71-86, 2003

FARIAS, $M$ et al . Caracterização das notificações de violência em crianças no município de Ribeirão Preto, São Paulo, no período 2006-2008. Epidemiol. Serv. Saúde, Brasília, v. 25, n. 4, p. 799-806, dez. 2016. Disponível em http://www. scielo.br/scielo.php?script=sci_arttext\&pid=S2237-96222016000400799\&lng=pt\&nr m=iso . acesso em 23 jan. 2018.

HABIGZANG, L. F; KOLLER, S.H; AZEVEDO, G.A.; MACHADO, P.X. Abuso sexual infantil e dinâmica familiar: aspectos observados em processos jurídicos. Psicologia: Teoria e Pesquisa, v.21, n.3,p. 341-348, 2005.

IPEA; FBSP. Atlas da Violência 2018. 92 fls. Daniel Cerqueira -(Pesquisador/coord). Rio Janeiro: Ipea - Instituto de Pesquisa Econômica Aplicada \& FBP- Forum Brasileiro de Segurança Pública, 2018. 
MELO, A; SILVA, G.D; GARCIA, .P. Mortalidade de homens jovens por agressões no Brasil, 2010-2014: estudo ecológico. Cad. Saúde Pública, Rio de Janeiro, v. 33, n. 11, :e00168316 2017 Disponível em: http://www.scielo.br/scielo.php?script=sci_arttext\&pid=S0102-311X2017001105008\&lng=en\&nrm=iso . Acesso 20 jan. 2018.

MINAYO, M C S. Violência contra crianças e adolescentes: questão social, questão de saúde. Rev. Bras. Saude Mater. Infant., Recife, v. 1, n. 2, p. 91-102, Aug. 2001 . Disponível em: http://www.scielo.br/scielo.php?script=sci_arttext\&pid=S1519-.38292001000200002\&lng=en\&nrm=iso. acesso 25 Nov. 2017.

OMS - Organização Mundial de Saúde. Relatório mundial sobre a prevenção da violência 2014. 275 fls. Organização Mundial de Saúde. Tradução e Revisão língua portuguesa NEV Núcleo de Estudos da Violência da Universidade de São Paulo , 2015

PERES, MFT; RUOTTI,C; CARVALHO, D. E REGINA, F.L. Vitimização fatal de crianças no espaço publico em decorrência da violência interpessoal comunitária: um diagnostico da magnitude e contextos de vulnerabilidade na America Latina. Rev. bras. segur. pública | São Paulo, v. 9, n. 2, p. 12-48, Ago/Set, 2015

PINTO JUNIOR, A.A; CASSEPP-BORGES, V; SANTOS, J.G. Perfil e estratégias de intervenção em violência doméstica infanto-juvenil . Cad. Saúde Colet., Rio de Janeiro, v.23, n.2, p. 124-131, 2015

REIS, D.; BARROS, A.Al S; CAVALCANTE, L.I. C. Agressor sexual de crianças e adolescentes: uma discussão sobre o gênero dos participantes na literatura. Psicol. rev. (Belo Horizonte), Belo Horizonte, v. 21, n. 2, p. 252-272, ago. 2015 - Disponível em: http://pepsic.bvsalud.org/scielo.php?script=sci_arttext\&pid=S1677-11682015000200004\&lng=pt\&nrm=iso. acesso em 26 nov. 2017.

SAFFIOTI, H.I.B. No Fio da Navalha: Violência Contra Crianças e Adolescentes no Brasil Atual. In: F.R MADEIRA (Org.) Quem Mandou Nascer Mulher? p. 134-211, São Paulo: Editora Rosa dos Tempos, 1997.

SANTOS CA, COSTA MCO, AMARAL MTR, NASCIMENTO SOBRINHO CL, MUSSE JO, COSTA AM. Agressor sexual de crianças e adolescentes: análise de situações relacionadas à violação e vítimas. Adolesc Saude. V.12, n.3 p.7-20, 2015;

UNICEF As ameaças à sobrevizência e ao desenvolvimento infantil. Em: Unicef em ação na América Latina e no Caribe. Disponível em: https://www.unicef.org/brazil/pt/ overview_9856.html. Acesso em 22 jan 2018.

UNODC. Global Study on Homicide 2013: trends, contexts, data. Viena: United Nations Office on Drugs and Crime, 2013. eISBN: 978-92-1-054205-0. Disponível em: https:// www.unodc.org/documents/data-and-analysis/statistics/GSH2013/2014_GLOBAL_ HOMICIDE_BOOK_web.pdf. Acesso em 02 dez 2017. 
Contato dos autores: mariliamartinsvizzotto@gmail.com

Texto recebido em 12/12/2017

Aceito em: 110/06/2018 